\title{
Gaze Direction Modulates the Relation between Neural Responses to Faces and Visual Awareness
}

\author{
Apoorva Rajiv Madipakkam, ${ }^{1,2,3}$ Marcus Rothkirch, ${ }^{1,3}$ @Matthias Guggenmos, ${ }^{1,3,4}$ Andreas Heinz, ${ }^{1,3}$ \\ and Philipp Sterzer ${ }^{1,3,4}$ \\ ${ }^{1}$ Department of Psychiatry, ${ }^{2}$ International Graduate Program Medical Neurosciences, and ${ }^{3}$ Berlin Center for Advanced Neuroimaging, Charité, \\ Universitätsmedizin Berlin, 10117 Berlin, Germany, and ${ }^{4}$ Bernstein Center for Computational Neuroscience, Humboldt-Universität zu Berlin, 10115 Berlin, \\ Germany
}

Gaze direction and especially direct gaze is a powerful nonverbal cue that plays an important role in social interactions. Here we studied the neural mechanisms underlying the privileged access of direct gaze to visual awareness. We performed functional magnetic resonance imaging in healthy human volunteers who were exposed to faces with direct or averted gaze under continuous flash suppression, thereby manipulating their awareness of the faces. A gaze processing network comprising fusiform face area (FFA), superior temporal sulcus, amygdala, and intraparietal sulcus showed overall reduced neural responses when participants reported to be unaware of the faces. Interestingly, direct gaze elicited greater responses than averted gaze when participants were aware of the faces, but smaller responses when they were unaware. Additional between-subject correlation and single-trial analyses indicated that this pattern of results was due to a modulation of the relationship between neural responses and awareness by gaze direction: with increasing neural activation in the FFA, direct-gaze faces entered awareness more readily than averted-gaze faces. These findings suggest that for direct gaze, lower levels of neural activity are sufficient to give rise to awareness than for averted gaze, thus providing a neural basis for privileged access of direct gaze to awareness.

Key words: consciousness; CFS; face processing; gaze direction

\section{Significance Statement}

Another person's eye gaze directed at oneself is a powerful social signal acting as a catalyst for further communication. Here, we studied the neural mechanisms underlying the prioritized access of direct gaze to visual awareness in healthy human volunteers and show that with increasing neural activation, direct-gaze faces enter awareness more readily than averted-gaze faces. This suggests that for a socially highly relevant cue like direct gaze, lower levels of neural activity are sufficient to give rise to awareness compared with averted gaze, possibly because the human brain is attuned to the efficient neural processing of direct gaze due to the biological importance of eye contact for social interactions.

\section{Introduction}

From everyday experience, we know that other people's eye gaze is an important nonverbal cue in social interactions. The direction of eye-gaze is pivotal to any social interaction by contributing information about another's intentions and focus of interest

Received March 2, 2015; revised July 15, 2015; accepted Aug. 5, 2015.

Author contributions: A.R.M., M.R., M.G., A.H., and P.S. designed research; A.R.M. and M.R. performed research; A.R.M., M.R., M.G., and P.S. analyzed data; A.R.M., M.R., M.G., A.H., and P.S. wrote the paper.

This work was supported by a grant from the German Research Foundation (DFG) to P.S. (STE 1430/2-1 and STE 1430/7-1); A.R.M. was supported by the DFG Cluster of Excellence Neurocure (EXC 257); and M.G. was supported by the DFG Research Training Group GRK1589/1.

The authors declare no competing financial interests.

Correspondence should be addressed to Apoorva Rajiv Madipakkam, Department of Psychiatry, Charité, Universitätsmedizin Berlin, Charitéplatz 1, 10117 Berlin, Germany. E-mail: apoorva-rajiv.madipakkam@charite.de.

DOI:10.1523/JNEUROSCI.0815-15.2015

Copyright $\odot 2015$ the authors $\quad 0270-6474 / 15 / 3513287-13 \$ 15.00 / 0$
(Kleinke, 1986; Emery, 2000). The paramount importance of eyegaze processing for social functioning is highlighted by the fact that sensitivity to eye-gaze information is altered in psychiatric disorders that are characterized by impairments in social interaction, such as autism or social phobia (Horley et al., 2003; Senju et al., 2005).

Direct gaze is a social cue of particularly high relevance, as it often represents the first step in social interactions, indicating either approach or avoidance behavior. It is also thought to be a form of social reward, activating central reward systems more strongly than an averted gaze (Kampe et al., 2001). The high priority we give specifically to direct gaze is substantiated by a large body of research and is referred to as the "eye contact effect" (Senju and Johnson, 2009). Direct gaze facilitates a variety of high-level cognitive functions, such as face recognition and 
memory (Mason et al., 2004), gender discrimination (Macrae et al., 2002), and decision making in social contexts (Snyder et al., 1974; Kleinke and Singer, 1979). There is also evidence for directgaze effects on lower-level sensory processes, such as involuntary capture and cuing of attention (von Grünau and Anston, 1995; Senju and Hasegawa, 2005; Conty et al., 2006). Notably, humans' sensitivity to gaze information is not restricted to situations in which others' faces are clearly visible. For instance, attention is influenced by the direction of eye gaze even when faces are presented subliminally (Sato et al., 2007; Rothkirch et al., 2015). Importantly, it has repeatedly been shown that direct gaze rendered invisible by interocular suppression gains access to awareness faster than averted gaze (Stein et al., 2011; Chen and Yeh, 2012), suggesting that the human brain is attuned to the fast and efficient detection of direct gaze.

The neural processing of eye gaze direction involves a specialized system that comprises occipitotemporal lobe regions like the fusiform face area (FFA), superior temporal sulcus (STS), and the amygdala, as well as the intraparietal sulcus (IPS; Haxby et al., 2002; Nummenmaa and Calder, 2009). Recent studies using functional magnetic resonance imaging (fMRI) and electroencephalography (EEG) have shown differential neural responses to eye-gaze directions even in the absence of awareness (Burra et al., 2013; Yokoyama et al., 2013). Although there is thus evidence for neural gaze processing with and without awareness, the neural mechanisms underlying the prioritized access of direct gaze to awareness are still unknown. Due to the central role that eye gaze and especially direct gaze plays in nonverbal social communication, insights into the neural basis of its privileged access to awareness are an important prerequisite for a more comprehensive understanding of the processes that govern human social interactions.

Here, we performed an fMRI study using an interocular suppression technique, continuous flash suppression (CFS; Tsuchiya and Koch, 2005), to manipulate participants' awareness of visual face stimuli. CFS has proven to be a useful method to investigate the potency of visual stimuli to gain access to awareness (Gayet et al., 2014; Stein and Sterzer, 2014). We therefore used CFS to manipulate awareness in a controlled way and to measure neural responses in direct relation to the privileged access of direct gaze to visual awareness. Photographs of faces with either direct or averted gaze were presented to one eye while the other eye was concurrently presented with high-contrast dynamic mask stimuli. Participants indicated their awareness of the face stimuli after each trial. Critically, the contrast of the face stimuli was kept constant throughout the experiment, thus allowing us to determine the relationship between neural responses to direct and averted gaze, respectively, and visual awareness of the face stimuli.

\section{Materials and Methods}

\section{Participants}

Twenty-one participants volunteered for the study. The data from two participants were discarded from all analyses, one due to excessive head motion during fMRI measurements, exceeding an a priori defined motion threshold of $3 \mathrm{~mm}$ within a run, and the other one due to incomplete data collection as the participant aborted the experiment. Three further participants were excluded due to methodological reasons related to data analysis (see Analysis of behavioral data). The final sample consisted of 16 participants ( 13 females) with a mean age of 25 years (SEM: 0.74 years). All participants had normal or corrected-to-normal vision, were naive to the purpose of the study, and were paid for their participation. The study was approved by the local ethics committee and written informed consent was obtained from all participants before the experiment.

\section{Stimuli}

We used three different identities of greyscale faces with neutral expression that had also been used in previous studies investigating gaze direction (Senju and Hasegawa, 2005; Senju et al., 2005; Stein et al., 2011). The stimuli had laterally averted heads, and the iris and pupils were shifted horizontally within the eyes to give the impression of eye gaze being either directed at the observer (when eye gaze was oriented opposite to the head direction) or averted from the observer (when eye gaze and head were oriented in the same direction; Fig. $1 A$ ).

Thus, stimulus differences between the two gaze directions were minimized and only related to the eye region. The stimuli were cropped to oval shapes $\left(3.6^{\circ} \times 4.4^{\circ}\right.$ of visual angle $)$ and equalized for global contrast (root mean square contrast of 0.05) and luminance. Visual stimuli were presented with MATLAB (MathWorks), using the Cogent 2000 toolbox (http://www.vislab.ucl.ac.uk/cogent.php). The visual masks used for CFS (see Design and procedure) consisted of black, white, and gray circles (sizes ranging from $0.01^{\circ}$ to $0.97^{\circ}$ ) constituting a square of $12^{\circ}(\mathrm{Mi}-$ chelson contrast: 1 ) and were flashed at a frequency of $10 \mathrm{~Hz}$. In the fMRI scanner, the stimuli were projected via an LCD projector (ProExtra Multiverse Projector, Sanyo Electric; refresh rate $60 \mathrm{~Hz}$ ) onto a screen of size $24.9^{\circ} \times 18.6^{\circ}$. Dichoptic presentation of the stimuli was achieved using an fMRI compatible cardboard divider and a pair of prism lenses that were worn by the participants (Schurger, 2009).

\section{Design and procedure}

Before the start of the fMRI experiment, each participant's dominant eye was identified in an eye dominance test. Both the fMRI experiment and eye dominance test used CFS as an interocular suppression technique. CFS manipulates observers' awareness of visual stimuli such that stimuli can be suppressed from awareness for prolonged periods of time. It has proven to be a useful method to investigate the ability of visual stimuli to gain access to awareness (Gayet et al., 2014; Stein and Sterzer, 2014). While one eye is presented with the stimulus of interest, the other eye is presented with high-frequency dynamic mask images. Initially, the static stimulus is suppressed from awareness by the mask stimuli; after a variable period of time the static stimulus usually overcomes suppression and is consciously perceived by the observer. Using CFS to suppress stimuli from awareness allowed us to relate our neural findings to the previously well established behavioral observations where direct gaze has been shown to overcome suppression by CFS faster than averted gaze (Stein et al., 2011; Chen and Yeh, 2012). We could therefore establish a direct link between neural activity and access to awareness.

In the eye dominance test, the face stimuli described above (see Stimuli) were presented in one of the quadrants of a white square $\left(5^{\circ} \times 5^{\circ}\right)$ to one eye, while simultaneously CFS masks consisting of black and white circles were flashed at a frequency of $10 \mathrm{~Hz}$ to the other eye. Participants were required to indicate the location of the face by button press as soon as any part of the face became visible, and to respond as fast and as accurately as possible. The presentation of the face stimuli to the two eyes was randomized across trials. Reaction times, that is, the time until participants indicated awareness of the face, were averaged for each eye separately. The eye at which the face stimuli were on average detected faster was defined as the participant's dominant eye.

In the fMRI experiment, face stimuli were presented for a fixed period of time and participants indicated their level of awareness after stimulus presentation. Each trial started with a prestimulus fixation period (jittered randomly between 2 and $4 \mathrm{~s})$ during which a white frame $\left(12^{\circ} \times\right.$ $\left.12^{\circ}\right)$ and a central blue fixation cross $\left(0.6^{\circ} \times 0.6^{\circ}\right)$ were presented on a gray background $\left(30 \mathrm{~cd} / \mathrm{m}^{2}\right)$ to both eyes (Fig. $1 B$ ). Then, a face stimulus with one of the two gaze directions was presented intermittently to the participant's nondominant eye while concurrently CFS masks consisting of black and white circles were flashed at a frequency of $10 \mathrm{~Hz}$ to the dominant eye. The face was presented either left or right of fixation on the horizontal meridian at an eccentricity of $3.4^{\circ}$. The stimulus presentation consisted of six repetitions of the following sequence. First, face and mask stimuli were presented for $800 \mathrm{~ms}$. Second, mask stimuli were 
A
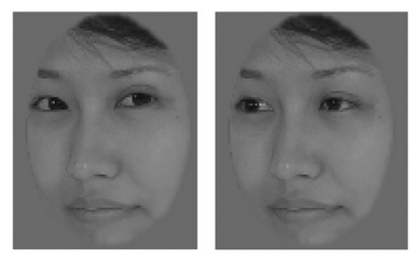

B

CFS trial

non-dom. dominant eye eye

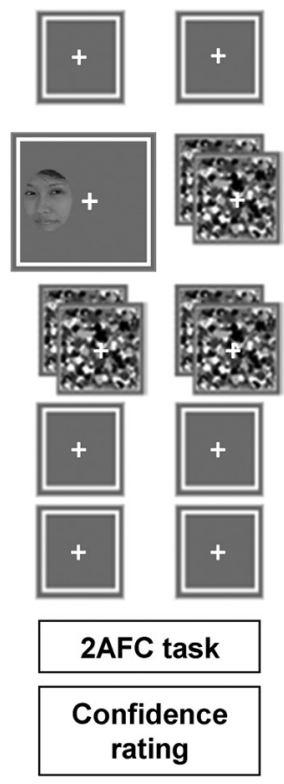

No-CFS trial Both
eyes
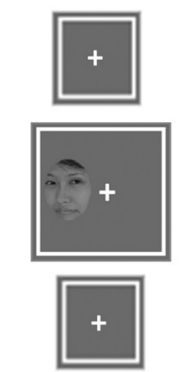

2AFC task

Confidence rating

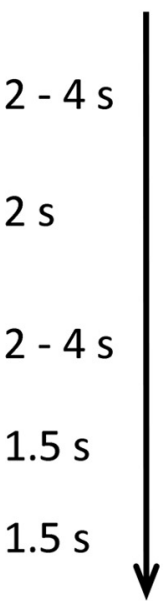

$2-4 s$

$1.5 \mathrm{~s}$

$1.5 \mathrm{~s}$

of visual stimuli (Zehetleitner and Rausch, 2013). They therefore allowed us to assign a level of awareness to each respective trial. Participants were thus considered to be aware or unaware of the stimulus based only on their subjective reports in confidence (Szczepanowski et al., 2013). It is important to note that participants were explicitly instructed to report the location of the face and not anything else they thought they might have perceived. Because the stimulus conditions and mask contrasts were constant throughout the experiment, the only systematic stimulus difference between trials was the gaze direction of the face stimuli.

In addition to CFS trials, which were of primary interest for our research question, the fMRI experiment also included no-CFS trials, in which faces were presented simultaneously to both eyes without a CFS mask and were thus fully visible. Of note, the no-CFS trials were included as a control condition to verify activation in our regions-of-interest (ROIs) by the face stimuli when they were clearly visible. In the no-CFS trials, the face stimulus with either direct or averted gaze was presented once for $2 \mathrm{~s}$ to both eyes. All other prestimulus and poststimulus parameters with respect to fixation timings and the task were the same as in CFS trials. For both CFS and no-CFS trials, the order of side of presentation and faces' gaze directions was counterbalanced and randomized across trials for each participant.

In total, the scanning session lasted for $\sim 60$ min and consisted of 10 runs with 24 trials each (12 CFS trials +12 no-CFS trials). Participants were instructed to maintain fixation on the central cross throughout the task. To aid fixation, the blue fixation cross was presented throughout the whole trial in both CFS and no-CFS trials, that is, also on top of the masks in the CFS trials. Eye movements were monitored online and analyzed off-line using an MRI compatible, infrared video eye tracker with a sampling rate of 50 $\mathrm{Hz}$ (SMI iVIEW X MRI-LR, SensoMotoric Instruments) to ensure that participants were able to hold fixation.

After the main experiment, an additional

presented to both eyes for $200 \mathrm{~ms}$. Finally, only the central fixation cross was shown along with the white frame for $500 \mathrm{~ms}$. The sixth repetition of this sequence was followed by a poststimulus fixation period jittered randomly between 2 and $4 \mathrm{~s}$.

Such a design of intermittent stimulus presentation has been used in previous fMRI studies (Sterzer et al., 2008; Hesselmann et al., 2011) investigating the neural responses to stimuli rendered invisible using CFS. The rationale for this procedure is based on the observation that intermittent stimulus presentation stabilizes perceptual states during binocular rivalry, which forms the basis for CFS (Leopold et al., 2002; Pearson and Brascamp, 2008; Sterzer and Rees, 2008). In other words, the aim of intermittent stimulus presentation was to minimize perceptual alterations between the face and mask stimuli and to increase the perceptual dominance of the masks. Participants were then prompted with two questions, each presented for $1.5 \mathrm{~s}$, to which they responded using button presses. The first question was a spatial two-alternative forced choice (2AFC) task in which participants had to report whether the face was presented to the left or the right of the fixation cross. This was followed by a rating of their confidence in having correctly guessed the face stimulus location. The confidence rating scale was a 4-point scale with four response options (very sure, sure, unsure, and highly unsure). Confidence ratings are the most frequently used subjective measure of awareness and have been shown to provide a good reflection of participants' awareness functional localizer scan was performed to individually identify the most face-responsive voxels in the brain. The localizer consisted of 20 alternating blocks of greyscale face and house stimuli presented foveally. The duration of each block was $13.6 \mathrm{~s}$, during which 12 stimuli of the same category were presented for $560 \mathrm{~ms}$ with an inter trial interval of $560 \mathrm{~ms}$. Participants performed a one-back task on the face and house stimuli. A rest period of $9 \mathrm{~s}$ followed each block of stimulus presentation leading to a total duration of $7.5 \mathrm{~min}$ for the localizer scan sequence.

\section{fMRI acquisition}

Functional $\mathrm{T} 2{ }^{*}$-weighted MR images were acquired on a TRIO 3T scanner (Siemens) equipped with a 12-channel head coil using an echo planar imaging sequence (echo time: $25 \mathrm{~ms}$, repetition time: $2.12 \mathrm{~s}$, flip angle: $78^{\circ}$ ). Whole-brain coverage was obtained with 38 contiguous slices (voxel size: $3 \times 3 \times 3 \mathrm{~mm}^{3}$ ), oriented parallel to the anterior-posterior commissure line. For the main experiment, 167 volumes were obtained in each run, which had a duration of $\sim 5.9$ min. Additionally T1weighted structural images (MPRAGE, voxel size $1 \times 1 \times 1 \mathrm{~mm}^{3}$ ) were acquired. Finally, the face localizer scan of 220 volumes was performed.

\section{Analysis of eye tracking data}

Eye tracking data of four participants could not be acquired due to calibration difficulties. For the remaining 12 participants, data were ac- 
quired in at least 7 of the 10 runs. Horizontal and vertical eye positions were collected throughout the whole experiment. Data were then analyzed based on the horizontal eye positions during the presentation of the face stimuli in both the CFS trials and no-CFS trials within a time window of $500 \mathrm{~ms}$ before face stimulus onset till $500 \mathrm{~ms}$ after offset of the face stimulus. After excluding artifacts, baseline correction, which was performed to remove inconsistencies that can arise due to the participants wearing prism lenses, was performed for each run separately. We defined the baseline as the mean of all horizontal eye positions across the run. Eye positions were then plotted for each participant and inspected visually for systematic eye movement differences between the gaze directions. To test whether the horizontal distributions of eye positions differed between gaze directions or awareness of the faces, for each participant, a Kolmogorov-Smirnov (KS) test was applied to the distribution of horizontal eye positions for the different trials (CFS/no-CFS) and gaze directions (direct/averted). Importantly, this comparison was not made on the means of all eye positions but on all data points recorded for each participant. However, as the KS test may be insensitive to subtle differences in eye movements, we additionally calculated the proportion of eye positions that landed on the stimulus region for each condition. This analysis was performed to further rule out the possibility that participants may have been biased to perform eye movements toward direct gaze, based on previous findings that direct gaze captures attention (Senju and Hasegawa, 2005; Rothkirch et al., 2015).

To assess the strength of the evidence for the null hypothesis, as a nonsignificant $t$ test result cannot provide unequivocal evidence in favor of the null hypothesis, we also performed a Bayes analysis (Dienes, 2011; Sterzer et al., 2014). This gives the likelihood of the data given the null hypothesis and the likelihood of the data given the alternative hypothesis and their quotient, the Bayes factor, as an output. Bayes factors $<0.33$ provide substantial evidence for the null over the alternative hypothesis, whereas Bayes factors $>3$ can be interpreted as evidence for the alternative over the null hypothesis (Dienes, 2011). The analysis was performed using an online Bayes calculator (http://www.lifesci.sussex.ac.uk/home/ Zoltan_Dienes/inference/bayes_factor.swf).

\section{Analysis of behavioral data}

CFS trials in which participants failed to give a response in either the spatial 2AFC task or the confidence rating were excluded from analyses ( $\mathrm{M}=2.6 \% \pm 0.57 \%$ SEM). Participants' awareness of the face stimuli was determined for each trial based on their respective subjective confidence report. For the main analysis of the fMRI data, we classified trials as unaware or aware. Aware trials comprised those in which participants indicated one of the three highest confidence levels (very sure, sure, and unsure). In contrast, in trials in which participants reported the lowest level of confidence (i.e., highly unsure), they were regarded as being unaware of the face stimuli. This classification of trials allowed us to be certain that the unaware trials only consisted of those in which participants expressed a complete absence of any subjective experience of the stimulus. The aware trials, in contrast, comprised all other levels of confidence, indicating that participants had at least some form of subjective experience of the face stimuli. Participants' performance in the 2AFC task for each confidence rating was then determined to confirm that their subjective experience of the stimulus was indeed reflected in their ability to perform the task, that is, providing support for participants' ability to distinguish between graded levels of awareness.

The proportion of direct and averted gaze trials in the "aware" and "unaware" categories was computed for each participant. Three participants had to be excluded as there were an insufficient number of CFS trials $(<10 \%)$ in either of the categories, aware or unaware. This resulted in a final sample of 16 participants.

\section{fMRI data analysis}

fMRI data were analyzed using SPM8 (http://www.fil.ion.ucl.ac.uk/spm/ software/spm8/). Functional images from the main experiment and the face localizer were slice-time-corrected and realigned to the first image to correct for head motion. After realignment, each participant's structural T1 image was coregistered to the first functional image. Coregistered structural T1 images and functional images were then segmented and spatially normalized to MNI space. In the final step, all normalized images were spatially smoothed with an $8 \mathrm{~mm}$ full-width at half-maximum Gaussian kernel.

Statistical inference: neural effects of gaze direction. The fMRI data from the main experiment and the face localizer were analyzed voxelwise in the context of the general linear model (GLM) approach. In a first step, GLMs were estimated for each participant. A high-pass filter with a $128 \mathrm{~s}$ cutoff was used to remove low-frequency fluctuations and temporal autocorrelations were corrected with an autoregressive model of order one $[\mathrm{AR}(1)+$ white noise]. Six regressors of interest were included in the GLM. These included two regressors for direct and averted gaze in the no-CFS trials. The remaining four regressors represented CFS trials with direct and averted gaze that were either categorized as aware or unaware based on the participants' confidence ratings. All regressors of interest were modeled as boxcar functions convolved with the canonical hemodynamic response function as implemented in SPM8. Two regressors representing the button presses of participants' responses to the $2 \mathrm{AFC}$ task and confidence rating were modeled as stick functions and incorporated into the GLM as regressors of no interest along with the six motion parameters produced by the realignment procedure. In addition, we ran a second model to analyze how neural responses covary with participants' level of awareness in a trialwise manner. To this end, we modeled each single trial in the CFS condition with a separate regressor. Separate regressors therefore resulted in one parameter estimate for each CFS trial. As each CFS trial was also associated with a subjective confidence rating, we could analyze the trialwise relationship between the neural responses to gaze direction and visual awareness. Furthermore, two regressors for direct and averted gaze in the no-CFS trials, two regressors for the participants' responses to the $2 \mathrm{AFC}$ task and confidence rating, as well as six motion parameters similar to the first model were included as regressors of no interest. Data from the face localizer run were analyzed analogously using the GLM approach in SPM8 and the two regressors of interest (faces vs houses) were modeled as boxcar functions convolved with the hemodynamic response function.

Model specification and estimation was then followed by an estimation of T-contrasts of stimulus-related responses at each voxel for each regressor of interest. This was determined using multiple linear regressions and scaled to the global mean signal of each run across conditions and voxels. For the localizer, a T-contrast was used to identify voxels that responded more strongly to the presentation of faces versus houses.

Our analyses focused on brain regions that are known to be critically involved in the processing of clearly visible eye gaze, namely the FFA (George et al., 2001), amygdala (Kawashima et al., 1999; Straube et al., 2010; Burra et al., 2013; Sauer et al., 2014), STS (Engell and Haxby, 2007; Materna et al., 2008), and IPS (Hoffman and Haxby, 2000). We identified the most face-responsive voxel within each ROI for each participant individually. To this end, the voxel with the peak activation in the $T$ contrast of faces $>$ houses in the functional face localizer scan was determined for each ROI in the two hemispheres. ROIs were defined by anatomical or publication-based probabilistic masks. For the FFA [mean peak voxel left: $(x, y, z)=-41,-54,-19$; right: $(x, y, z)=43,-53,-18]$ and the amygdala [mean peak voxel left: $(x, y, z)=-22,-4,-15$; right: $(x, y, z)=22,-6,-14]$, we used anatomical masks of the fusiform gyrus and the amygdala, respectively, provided by the WFU pickatlas (http:// fmri.wfubmc.edu/software/PickAtlas). To study the neural responses in the STS [mean peak voxel left: $(x, y, z)=-49,-56,13$; right: $(x, y, z)=$ $52,-54,10]$ and IPS [mean peak voxel left: $(x, y, z)=-35,-60,45$; right: $(x, y, z)=39,-53,50]$, probabilistic masks for these regions were generated based on coordinates obtained from at least nine previous studies investigating facial stimuli and by fitting a 3D Gaussian model to the coordinate set (STS: Puce et al., 1998; Hoffman and Haxby, 2000; George et al., 2001; Hooker et al., 2003; Pelphrey et al., 2004, 2005; Mosconi et al., 2005; Schilbach et al., 2006; Engell and Haxby, 2007; Sato et al., 2008, 2009; Nummenmaa et al., 2010, 2012; Carlin et al., 2011; Ethofer et al., 2011; IPS: Hoffman and Haxby, 2000; Kato et al., 2001; Hooker et al., 2003; Grosbras et al., 2005; Bristow et al., 2007; Hadjikhani et al., 2008; Nummenmaa et al., 2010, 2012). We then extracted parameter estimates from the individually defined peak voxels in all our ROIs from each hemisphere for the six regressors of interest. Please note that, 
A

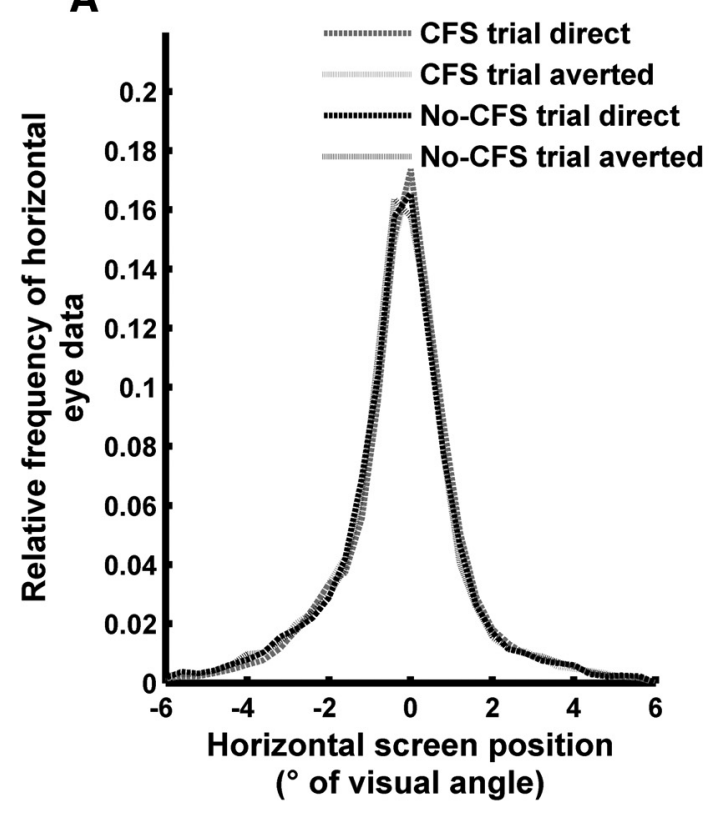

B

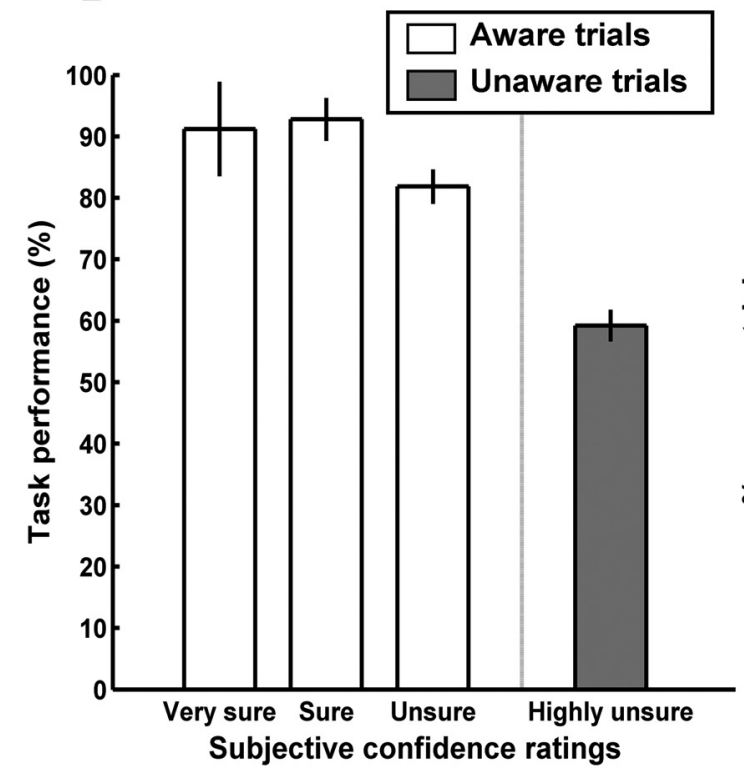

C

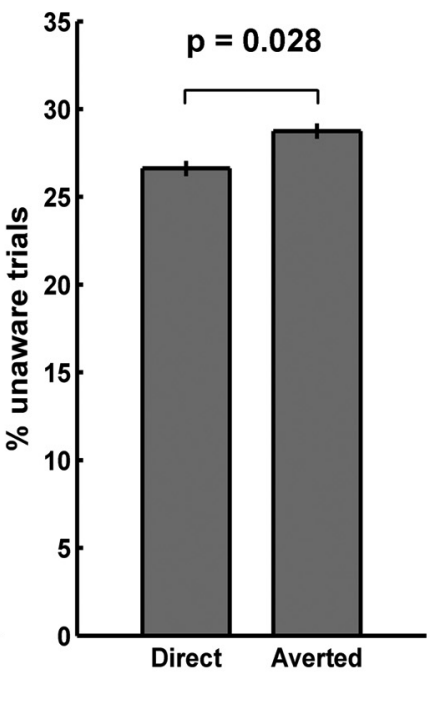

Figure 2. A, Distribution of horizontal eye positions during the presentation of the face stimuli with direct and averted gaze directions during the CFS and no-CFS trials in the fMRI experiment across all participants showing that fixation was maintained throughout the task. The face stimuli were presented at an eccentricity of $3.4^{\circ}$. B, Performance in the $2 \mathrm{AFC}$ task at each confidence rating. All trials in the lowest confidence rating highly unsure were considered unaware, whereas trials belonging to the first three ratings were considered aware for all analyses. C, Percentages of trials with direct and averted gaze, respectively, that remained unaware during CFS, relative to the total number of trials. A significantly smaller number of trials with direct gaze remained unaware compared with trials containing a face with averted gaze. Error bars denote within-subject SEM (Cousineau, 2005).

due to spatial smoothing, the peak voxel in each ROI represents a weighted average of all voxels within the smoothing kernel. It is also important to note that negative parameter estimates do not indicate a deactivation, but rather reduced neural signals in this voxel compared with the mean signal in this voxel across the whole time course.

For statistical inference, parameter estimates from each ROI were subjected to separate $2 \times 2 \times 2$ repeated-measures ANOVA (rmANOVA) with the factors awareness (aware/unaware), hemisphere (left/right), and gaze direction (direct/averted) using the statistical software package SPSS 19. The main effects from the ANOVA were then Bonferroni-corrected for the number of ROIs (i.e., 4). Significant interaction effects were fur- ther explored with post hoc $t$ tests that were Bonferroni-corrected for the number of comparisons (i.e., 2). $p_{\text {corr }}$ refers to the significance level after Bonferroni correction for multiple comparisons.

Correlational analysis between behavioral and neural responses to gaze. Behaviorally, a preferential access to awareness for direct versus averted gaze would be reflected in a smaller number of unaware direct-gaze trials than averted-gaze trials. To quantify such a preference for direct gaze for each participant, we computed a "behavioral direct-gaze index," which was defined as follows:

$$
\frac{n_{a}-n_{d}}{n_{a}+n_{d}} .
$$

$n_{a}$ indicates the number of unaware trials containing a face with averted gaze and $n_{d}$ indicates the number of unaware trials containing a face with direct gaze. Positive values signify a greater sensitivity to direct gaze. At the neural level, we defined a "neural direct-gaze index" by subtracting individual BOLD contrast estimates for unaware averted-gaze faces from the contrast estimates for direct-gaze faces. Here, increasing values indicate greater responses to direct-gaze relative to avertedgaze faces.

Trialwise analyses of neural responses to direct and averted gaze with respect to awareness. To investigate how the neural responses to gaze direction affects awareness of the face stimuli, we extracted parameter estimates from the second GLM, where each CFS trial was modeled as a separate regressor of interest. Thus, every single CFS trial was associated with a separate parameter estimate and a confidence rating. This trialwise analysis procedure therefore allowed us to relate the neural response to the participant's awareness level in a given trial. It is important to note that the major difference between this analysis and that using the first GLM (see Statistical inference: neural representation of gaze direction) is the trialwise assessment of the relationship between neural responses and awareness in the second GLM. This analysis was restricted to responses in the FFA, because neural signals in this region exhibited the most robust relation to participants' behavioral responses (see Results, Correlation analysis between behavioral and neural responses to gaze). The neural responses in the FFA were averaged across the left and right hemispheres, as no main effect of hemisphere was revealed in our main analysis (see Results, Neural responses to faces in CFS trials). The extracted parameter estimates for all CFS trials from the FFA were pooled across both gaze conditions and sorted into quartiles based on their magnitude. Because each trialwise parameter estimate was associated with a confidence rating, we could compute the average proportion of each confidence rating for direct and averted gaze for each quartile. A $2 \times 4$ rmANOVA with the factors gaze direction (direct/averted) and quartiles were performed for the proportion of unaware trials for statistical inference.

Exploratory whole-brain analyses. In addition to the ROI analyses described above, we also performed exploratory analyses with uncorrected significance thresholds ( $p<0.001$, uncorrected, cluster size 10 voxels) 
for the comparison of direct and averted gaze both in CFS trials and visible no-CFS trials.

\section{Results}

\section{Eye-tracking data}

The distribution of the horizontal eye data for the different conditions is shown in Figure 2A. A Kolmogorov-Smirnov test for the distributions of horizontal eye positions in the different conditions (direct and averted face stimuli in CFS and no-CFS trials) for each participant revealed no significant differences in eye movements (all $p$ values $>0.5$ ). Furthermore, the average proportion of eye positions that fell on the stimulus across all subjects was not significantly different between CFS direct-gaze trials $(\mathrm{M}=4.65 \% \pm 0.33 \% \mathrm{SEM})$ and $\mathrm{CFS}$ averted-gaze trials $(\mathrm{M}=$ $4.26 \% \pm 0.33 \%$ SEM; $\left.t_{(11)}=0.59, p=0.57\right)$. To assess the strength of the evidence for the null hypothesis we performed a Bayes analysis on a uniform distribution varying from 0 (no mean difference between conditions) and 100\% (maximum possible mean difference between conditions) to test that the mean differences between the conditions were not significantly different from each other. The analysis yielded a Bayes factor of 0.01 for the comparison between the CFS direct and averted-gaze, which can be interpreted as substantial evidence for the null hypothesis, that is, no differences in the proportions of eye movements toward direct versus averted gaze. Based on the above two analyses, we could safely conclude that participants were able to maintain central fixation equally well in all conditions.

\section{Behavioral data}

Participants' confidence ratings at the end of each trial were used as an index for their awareness of the face stimuli (Zehetleitner and Rausch, 2013). In approximately one-half of the trials, participants chose one of the first three levels of the confidence scale, indicating at least some degree of awareness of the faces (very sure: $\mathrm{M}=12.3 \% \pm 3.5 \%$ SEM; sure: $\mathrm{M}=10.1 \% \pm 1.6 \% \mathrm{SEM}$; unsure: $\mathrm{M}=20.4 \% \pm 2.2 \% \mathrm{SEM})$. In the remaining trials $(\mathrm{M}=$ $54.5 \% \pm 4.08 \%$ SEM), they expressed the lowest level of confidence ("highly unsure"). Based on these confidence ratings, we classified the first three confidence levels (i.e., "very sure," "sure," and "unsure") as aware trials and the last confidence level (i.e., highly unsure) as unaware trials. This classification into aware and unaware was corroborated by the $2 \mathrm{AFC}$ task performance for each of the four confidence categories (Fig. 2B). An rmANOVA with the factor confidence revealed a main effect of confidence $\left(F_{(3,36)}=18.22, p<0.001\right)$. Furthermore, Bonferroni-corrected post hoc $t$ tests between the different confidence ratings showed that the performance in all three types of aware trials was significantly higher than in the unaware (highly unsure) trials (very sure: $\left.t_{(12)}=4.27, p_{\text {corr }}<0.003\right)$; sure: $t_{(12)}=7.12, p_{\text {corr }}<0.001$; unsure $\left.t_{(12)}=5.83, p_{\text {corr }}<0.001\right)$. To probe whether task performance at the different levels of awareness was dependent on gaze direction, we performed a second rmANOVA including the factors gaze direction (direct/averted) and confidence. This analysis, however, showed no significant main effect of gaze or interaction effects $(p>0.2)$. The main effect of confidence still remained significant $\left(F_{(3,30)}=69.85, p<0.001\right)$.

Crucially, the percentage of trials that remained unaware during CFS differed between direct-gaze and averted-gaze trials (Fig. $2 C)$. Significantly less trials with direct gaze faces remained suppressed from awareness compared with the number of trials with averted gaze faces $\left(t_{(15)}=-2.43, p=0.028\right)$. This finding is in line with previous behavioral findings showing preferential ac- cess to awareness for direct versus averted gaze (Stein et al., 2011; Chen and Yeh, 2012).

\section{Neural responses to faces in CFS trials}

Next, we analyzed neural responses to face presentations in the CFS trials. Figure 3 shows the parameter estimates for the two gaze directions in aware and unaware trials that were extracted from the individual peak voxels in each ROI. For each ROI, these parameter estimates were subjected to a $2 \times 2 \times 2$ rmANOVA with the factors awareness (aware/unaware), hemisphere (left/ right), and gaze direction (direct/averted). This analysis revealed highly significant main effects of awareness $\left(\mathrm{FFA}: F_{(1,15)}=17.37\right.$, $p_{\text {corr }}=0.004$; amygdala: $F_{(1,15)}=17.58, p_{\text {corr }}=0.004 ;$ STS: $F_{(1,15)}=$ $11.28, p_{\text {corr }}=0.016$; IPS: $\left.F_{(1,15)}=14.52, p_{\text {corr }}=0.008\right)$, as well as awareness-by-gaze interactions in all four ROIs (FFA: $F_{(1,15)}=$ $26.71, p_{\text {corr }}<0.001$; amygdala: $F_{(1,15)}=13.93, p_{\text {corr }}=0.008 ;$ STS: $F_{(1,15)}=19.30, p_{\text {corr }}=0.004$; IPS: $\left.F_{(1,15)}=12.36, p_{\text {corr }}=0.012\right)$. There was no significant main effect of hemisphere (FFA: $F_{(1,15)}=$ $1.99, p_{\text {corr }}=0.72$; amygdala: $F_{(1,15)}<1$; STS: $F_{(1,15)}<1$; IPS: $\left.F_{(1,15)}<1\right)$ or interaction effects of hemisphere-by-awareness $\left(\right.$ FFA: $F_{(1,15)}=4.34, p_{\text {corr }}=0.22$; amygdala: $F_{(1,15)}<1$; STS: $F_{(1,15)}=2.51, p_{\text {corr }}=0.52$; IPS: $\left.F_{(1,15)}=3.62, p_{\text {corr }}=0.32\right)$, hemisphere-by-gaze $\left(\right.$ FFA: $F_{(1,15)}=1.60, p_{\text {corr }}=0.88$; amygdala: $F_{(1,15)}<1$; STS: $F_{(1,15)}=2.65, p_{\text {corr }}=0.48$; IPS: $\left.F_{(1,15)}<1\right)$, or three-way interaction of hemisphere-by-awareness-by-gaze (FFA: $F_{(1,15)}=1.82, p_{\text {corr }}=0.80 ;$ amygdala: $F_{(1,15)}=1.37, p_{\text {corr }}=1$; STS: $F_{(1,15)}<1$; IPS: $\left.F_{(1,15)}<1\right)$.

To explore the significant interaction effects of awareness and gaze, Bonferroni-corrected post hoc $t$ tests were computed to test for the differences between neural responses to direct versus averted gaze separately for aware and unaware trials across the left and right hemispheres. In line with the behavioral results implying a preferential processing of faces with direct gaze, we observed significantly larger neural responses to direct gaze in the FFA $\left(t_{(15)}=4.09, p_{\text {corr }}=0.002\right)$ and the IPS $\left(t_{(15)}=2.84, p_{\text {corr }}=\right.$ $0.024)$ when participants reported awareness of the faces. The STS showed a trend toward significance $\left(t_{(15)}=2.37, p_{\text {corr }}=\right.$ $0.064)$, whereas the amygdala did not show a significant difference between direct and averted gaze $\left(t_{(15)}=1.78, p_{\text {corr }}=\right.$ $0.18)$. Interestingly, and in contrast to the larger responses to direct gaze in aware trials, direct-gaze responses were significantly smaller than averted-gaze responses in the unaware trials in the amygdala $\left(t_{(15)}=-2.52 ; p_{\text {corr }}=0.048\right)$ and STS $\left.t_{(15)}=-3.73, p_{\text {corr }}=0.004\right)$. The FFA showed a trend toward significance after Bonferroni-correction $\left(t_{(15)}=-2.35, p_{\text {corr }}=\right.$ $0.064)$. There was a similar pattern in the IPS, which however, failed to reach significance $\left(t_{(15)}=-2.04, p_{\text {corr }}=0.12\right)$.

The significant interaction effect between awareness and gaze direction in all our ROIs, with larger neural responses to direct gaze faces when they were aware and smaller responses when they were unaware, could suggest that the regions of the gaze processing network are engaged in qualitatively different processes depending on the awareness of the critical face stimulus. Alternatively, because of their behavioral relevance, low neural responses may already be sufficient to promote awareness of faces with direct gaze, whereas in contrast, faces with averted gaze may still be suppressed from awareness at a similarly strong deployment of neural resources. To substantiate this latter notion, we next analyzed how gaze-related differences in access to awareness relate to neural responses to gaze directions in the absence of awareness. 

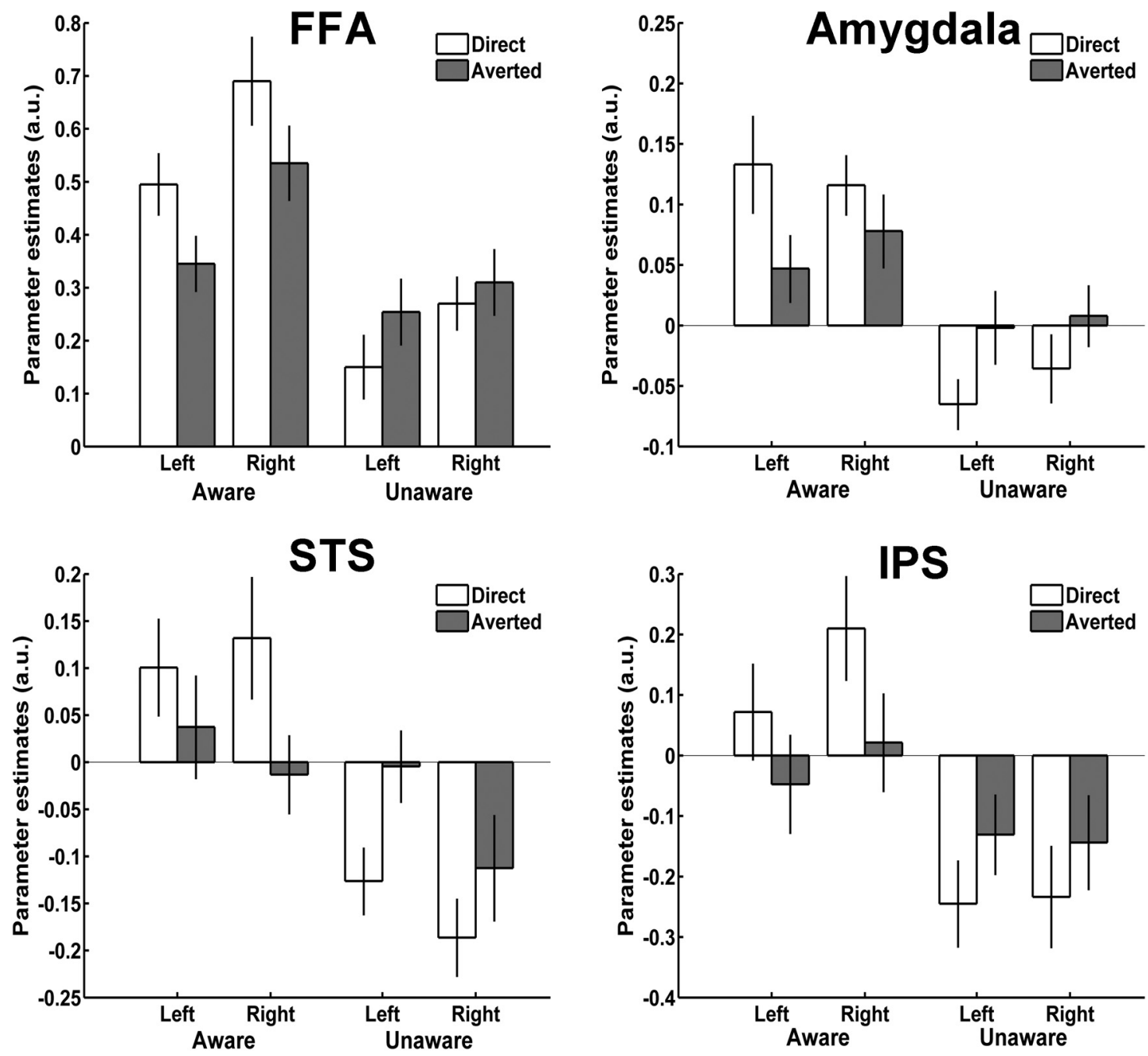

Figure 3. Neural responses to faces with direct and averted gaze direction presented along with CFS masks showing a significant main effect of awareness and interaction effect of awarenessby-gaze in all four regions of interest. Left and Right on the $x$-axes denote the hemispheres of the respective brain region. Error bars denote within-subject SEM (Cousineau, 2005).

Correlation analysis between behavioral and neural responses to gaze

We observed that: (1) participants became more readily aware of faces with direct gaze than of faces with averted gaze, and (2) faces with direct gaze elicited greater neural responses relative to faces with averted gaze, when participants were aware of the faces, and weaker responses, when they were unaware of the faces. To relate these behavioral and neural observations to each other, we tested for correlations between individual behavioral and neural directgaze indices in all our ROIs.

The behavioral direct-gaze index was defined as the difference between the number of unaware averted-gaze and direct-gaze trials divided by their sum. This index is thus a measure of the preferential access to awareness of direct versus averted gaze, where a positive behavioral index indicates a greater sensitivity toward direct gaze (that faces with direct gaze tend to reach awareness more readily than faces with an averted gaze). The neural direct-gaze index was computed as the difference in the BOLD response estimates between faces with direct and averted gaze in unaware CFS trials. Increasing neural indices therefore imply higher neural activity levels to unaware direct-gaze faces.

As there was no significant main effect of hemisphere in our analysis of neural responses to aware and unaware faces with

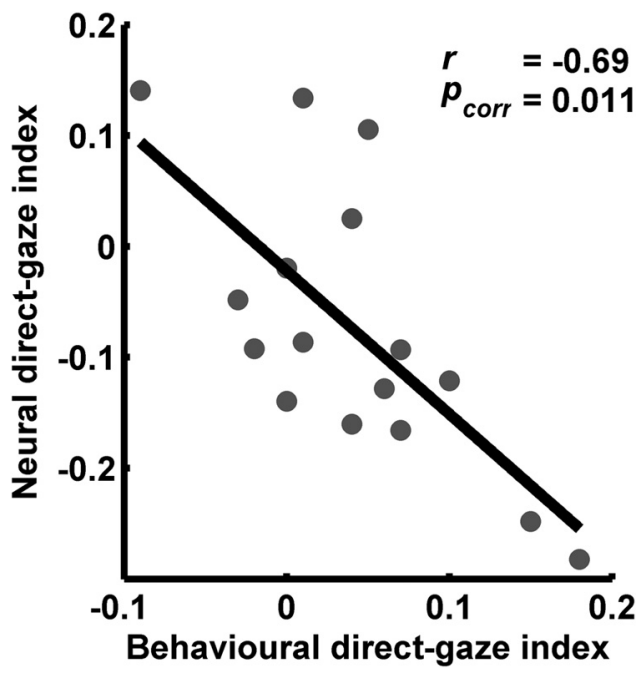

Figure 4. In the FFA, the behavioral direct-gaze index, where increasing values indicate increasing sensitivity to faces with direct gaze, correlated negatively with the neural direct-gaze index elicited by faces with direct gaze in the absence of awareness (relative to faces with averted gaze), suggesting that a strong behavioral index to direct gaze requires lower neural activity for these faces to become aware. 


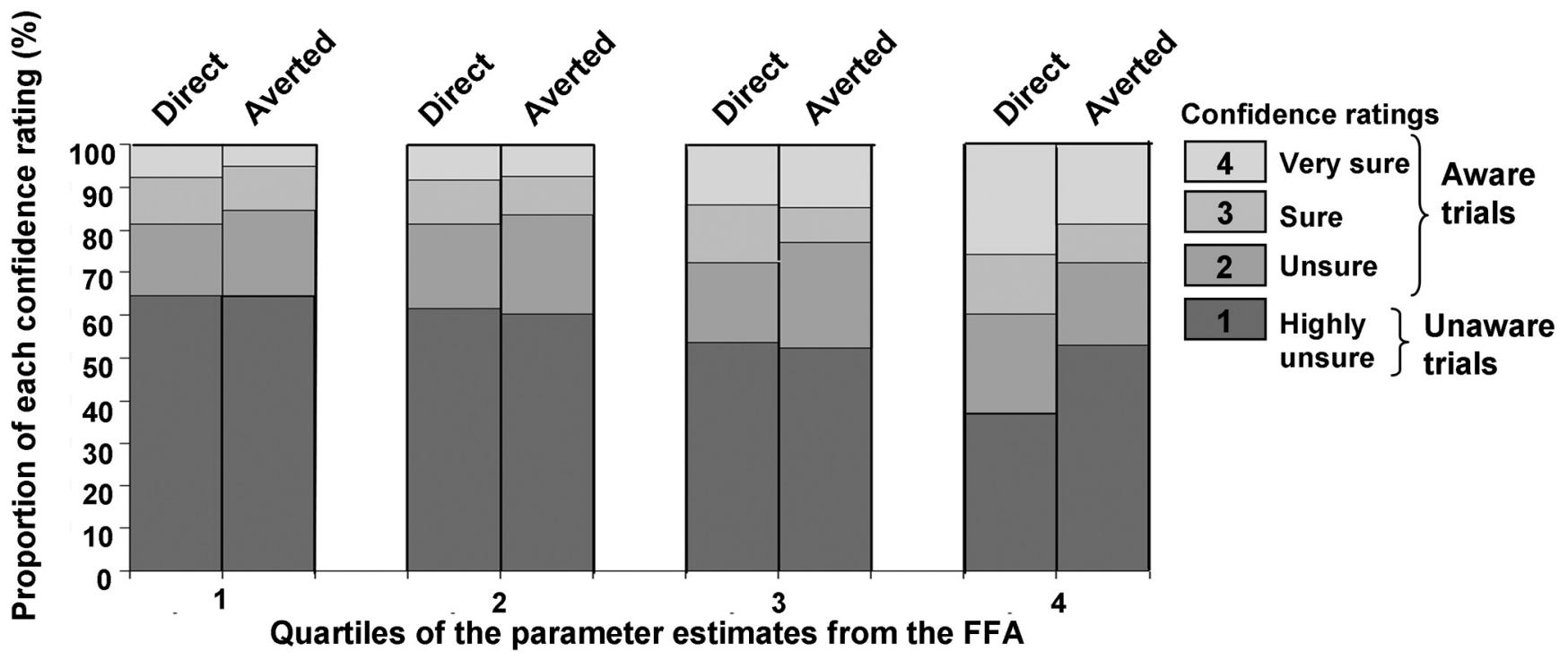

Figure 5. The mean proportion of each confidence rating is plotted for each quartile of the sorted trialwise parameter estimates for direct and averted gaze. In the fourth quartile, significantly fewer direct-gaze trials were rated the lowest confidence indicating that direct gaze has a lower threshold to access awareness compared with averted gaze.

direct gaze (see Neural responses to faces in CFS trials), correlations were performed for neural responses averaged across hemispheres. All ROIs showed strong negative correlations, but only the FFA (Fig. 4) exhibited a robust correlation that remained significant after Bonferroni correction for the number of ROIs $\left(r=-0.69, p_{\text {corr }}=0.011\right)$. The IPS showed a trend toward significance $\left(r=-0.57, p_{\text {corr }}=0.08\right)$. In amygdala and STS the correlations did not reach statistical significance (amygdala: $r=-0.52, p_{\text {corr }}=0.15$; STS: $r=-0.52, p_{\text {corr }}=$ $0.16)$.

The robust negative correlation between the behavioral index for direct gaze and the neural activity in the FFA shows that the individuals who had the greatest advantage for direct gaze to break into awareness, were those in whom we observed the greatest reduction of neural responses to unaware faces with direct gaze relative to unaware faces with averted gaze. This finding supports the interpretation that participants with a strong behavioral advantage for direct gaze became aware of these faces at lower neural activity levels compared to faces with an averted gaze. Accordingly, our group-level finding of relatively weaker FFA responses to unaware direct-gaze faces could reflect the fact that direct-gaze faces broke into awareness already at lower levels of FFA activity. Thus, only direct-gaze trials with relatively weak responses remained unaware, whereas direct-gaze trials with stronger responses, i.e., at the level of those in averted-gaze trials, were more likely to break interocular suppression and enter awareness. In contrast, faces with averted gaze may have required relatively more neural activity to break in to awareness, thus explaining their higher neural activity level in unaware trials.

Trialwise analysis of the relationship between neural responses and awareness

To corroborate the notion that direct-gaze faces reach awareness at lower levels of neural activity than averted-gaze faces, we further investigated the relationship between neural responses and awareness in a trialwise analysis. This analysis was performed for the FFA, where our between-subject correlation analysis had shown a significant association of neural responses and behavioral sensitivity. We estimated a second GLM where every CFS trial was modeled as a separate regressor, resulting in one beta estimate for each individual trial. All resulting beta estimates were sorted in quartiles according to their magnitude. As each parameter estimate was associated with a particular confidence rating in a given trial, we could compare the proportions of unaware direct-gaze and averted-gaze trials between quartiles. The mean proportion of each confidence rating for the two gaze directions in each quartile of the distribution of parameter estimates are depicted in Figure 5. A $2 \times 4$ rmANOVA for the proportion of unaware trials (trials rated with the lowest confidence, highly unsure) with the factors quartile and gaze (direct/averted) revealed a significant main effect of quartile $\left(F_{(3,45)}=10.10, p<\right.$ $0.001)$ and a significant gaze-by-quartile interaction effect $\left(F_{(3,45)}=4.82, p=0.005\right)$. The main effect of gaze was not significant $\left(F_{(1,15)}=3.03, p=0.10\right)$. Bonferroni-corrected post hoc $t$ tests (corrected for the number of tests, i.e., 4) showed that in the fourth quartile there were significantly less direct-gaze than averted-gaze trials that were unaware $\left(t_{(15)}=-4.21, p_{\text {corr }}=\right.$ $0.004)$. There was no difference between the proportions of unaware trials for direct and averted gaze in the other three quartiles (all $p>0.5$ ).

In the fourth quartile, where BOLD responses to direct and averted gaze were higher than in the first quartile, but still substantially lower than the average neural responses to fully visible faces (see Neural responses to faces in the no-CFS condition), there were markedly less unaware direct-gaze trials. Thus, at relatively high levels of BOLD responses (Fig. 5, fourth quartile), there is a greater advantage for direct gaze to access awareness compared with averted gaze (reflected by the fewer unaware trials). That is, at similar levels of BOLD responses, the probability that direct gaze reaches awareness is higher than that for averted gaze. This is in line with the result of our correlation analysis (see Correlation analysis between behavioral and neural responses to gaze) and provides further support for a gaze-dependent relationship between neural and behavioral responses. Thus, together these results suggest that faces with direct gaze require less neural activity than averted-gaze faces to overcome interocular suppression-or have a lower neural threshold for entering awareness. 
Table 1. Aware > unaware contrast for face stimuli across gaze directions

\begin{tabular}{llrrrrrr}
\hline & & \multicolumn{7}{l}{ Peak voxel coordinates } & & \\
\cline { 3 - 5 } Cerebral region & Laterality & $\mathrm{x}$ & $\mathrm{y}$ & $\mathrm{z}$ & & $\mathrm{N}$ & $p_{\text {FWE }}$ \\
\hline Insula & $\mathrm{L}$ & -30 & 29 & 1 & 8.39 & $<0.001$ \\
& $\mathrm{R}$ & 33 & 23 & -5 & 6.73 & $<0.001$ \\
Dorsolateral prefrontal cortex & $\mathrm{R}$ & 39 & 17 & 31 & 8.33 & $<0.001$ \\
Medial frontal gyrus & $\mathrm{L} / \mathrm{R}$ & -6 & 23 & 46 & 5.90 & 0.006 \\
Superior parietal cortex & $\mathrm{L}$ & -39 & -34 & 52 & 6.14 & 0.003 \\
Inferior parietal cortex & $\mathrm{R}$ & 36 & -46 & 46 & 6.10 & 0.003 \\
Intraparietal sulcus & $\mathrm{R}$ & 36 & -76 & 40 & 6.13 & 0.003 \\
\hline
\end{tabular}

\section{Whole-brain analysis}

Although neural responses in the gaze processing network were the focus of our study, we additionally performed whole-brain analyses to identify other brain regions involved in awarenessdependent processing of gaze direction. Even when lowering our significance threshold to an uncorrected level ( $p<0.001$, uncorrected), we neither observed any significant main effect for direct versus averted gaze nor interactions between awareness and gaze in brain regions outside our a priori ROIs. However, a significant main effect of awareness, i.e., stronger responses to aware versus unaware faces, was observed in several regions, which are listed in Table 1.

\section{Neural responses to faces in the no-CFS condition}

Finally, we analyzed the neural responses to fully visible faces in the no-CFS condition, which we had included as a control condition to verify face-related activation in our ROIs. For this purpose, parameter estimates from the individually defined peak voxels (Fig. 6) were subjected to $2 \times 2$ rmANOVAs with the factors hemisphere (left/right) and gaze direction (direct/ averted) for each ROI. This analysis yielded no main effects of gaze or interactions between gaze and hemisphere in any of the ROIs (all $p_{\text {corr }}$ values $>0.1$ ). However, a main effect of hemisphere was found in STS, indicating generally larger neural responses in the right hemisphere $\left(F_{(1,15)}=16.03, p_{\text {corr }}=0.001\right)$.

In addition, we also performed an exploratory univariate voxelwise whole-brain analysis. This did not reveal activation differences between direct and averted gaze after FWE correction and even after using a more liberal threshold of $p<0.001$ (uncorrected). We performed a further exploratory analysis for all voxels within our ROIs at an even more lenient threshold of $p<0.01$ for the contrasts of direct $>$ averted and averted $>$ direct. At this threshold, we found differential activation in bilateral IPS [right: $(x, y, z)=24,-73,46 ; p_{\text {uncorr }}=0.009$; left: $\left.(x, y, z)\right]=-24,-70$, $\left.34 ; p_{\text {uncorr }}=0.006\right]$, right STS $\left[(x, y, z)=60,-37,13 ; p_{\text {uncorr }}=\right.$ $0.001]$, and right amygdala $\left[(x, y, z)=24,-73,46 ; p_{\text {uncorr }}=\right.$ $0.001]$ for the contrast averted $>$ direct. The results should be interpreted with caution due to the liberal statistical threshold used, but are in line with previous studies showing the involvement of these regions in the monitoring and processing of eye gaze (Hooker et al., 2003; Pelphrey et al., 2003; Engell and Haxby, 2007; Sato et al., 2008).

\section{Analysis of neural responses to gaze in objectively unaware participants}

To rule out the possibility that any significant main effects or interactions were caused by participants' residual awareness of the face stimuli, we repeated all our analyses in participants' who were considered to be both subjectively unaware based on their confidence ratings and objectively unaware based on their performance in the 2AFC task. When performance in the 2AFC task was significantly above chance, based on a binomial test against $50 \%$, participants could not be considered objectively unaware of the stimuli and were excluded from this analysis. This exclusion criterion left us with a subset of 10 participants who had a mean performance of $52.75 \% \pm(1.6 \%$ SEM), which did not differ significantly from chance level of $50 \%$ (one-sample $t$ test: $t_{(9)}=$ $1.70, p=0.13$ ). We further performed a Bayes analysis on a uniform distribution varying from 0 to $50 \%$ and from 50 to $100 \%$ to test that the data were neither significantly above or below chance. The analysis yielded a Bayes factor of 0.32 and 0.02 for uniform distributions above and $<50 \%$, respectively, which can be interpreted as substantial evidence for the null hypothesis, that is, no significant difference from chance level.

Still however, similar to our analyses reported above (see Neural responses to faces in CFS trials), the neural responses in CFS trials with a $2 \times 2 \times 2$ rmANOVA with the factors awareness (aware/unaware), hemisphere (left/right), and gaze direction (direct/averted) revealed a significant main effect of awareness in the FFA $\left(F_{(1,9)}=10.37, p_{\text {corr }}=0.04\right)$ and $\operatorname{IPS}\left(F_{(1,9)}=9.95, p_{\text {corr }}\right.$ $=0.048)$, and an interaction effect of awareness-by-gaze $\left(F_{(1,9)}=\right.$ $\left.16.22, p_{\text {corr }}=0.012\right)$ in the FFA. Bonferroni-corrected post hoc $t$ tests for the interaction effect showed that neural responses to direct gaze $\left(t_{(9)}=3.16, p_{\text {corr }}=0.024\right)$ were significantly larger in aware trials. Direct-gaze responses although smaller than averted-gaze responses in the unaware trials, were not significant $\left.t_{(9)}=-2.08 ; p_{\text {corr }}=0.13\right)$. In the other ROIs, we also observed a similar main effect of awareness (amygdala: $F_{(1,9)}=6.16, p_{\text {corr }}=$ 0.14 ; STS: $\left.F_{(1,9)}=7.85, p_{\text {corr }}=0.08\right)$ and awareness-by-gaze interaction effects (amygdala: $F_{(1,9)}=8.73, p_{\text {corr }}=0.06$; STS: $F_{(1,9)}=$ $6.64, p_{\text {corr }}=0.12$; IPS: $\left.F_{(1,9)}=7.82, p_{\text {corr }}=0.08\right)$, but these effects were not robust enough to remain significant after Bonferroni correction. No other significant main effects or interactions were observed in any of the ROIs (all $p>0.1$ ).

Next, we performed the correlation between the neural and behavioral responses to gaze in this subset of 10 participants who were objectively unaware of the stimulus. Once again, we observed a strong negative correlation in the FFA $(r=-0.82$; $\left.p_{\text {corr }}=0.016\right)$. Although the IPS also had a strong negative correlation, it failed to reach significance after Bonferroni correction $\left(r=-0.64 ; p_{\text {corr }}=0.18\right)$. The negative correlations in the STS and amygdala were both insignificant (both $r<-0.39$; both $\left.p_{\text {corr }}>0.5\right)$.

Finally, the $2 \times 4$ rmANOVA of the single-trial analysis using the second GLM, for the proportion of unaware trials in each quartile in the FFA revealed a main effect of quartile $\left(F_{(3,27)}=\right.$ 11.27, $p<0.001)$ and a nearly significant interaction effect of gaze $\times$ quartile $\left(F_{(3,27)}=2.87, p=0.05\right)$. Post hoc $t$ tests for the proportion of unaware direct gaze trials in the fourth quartile was significantly less than the proportion of averted gaze trials $\left(t_{(9)}=\right.$ $\left.-3.7 ; p_{\text {corr }}=0.02\right)$. The proportion of unaware direct and averted gaze trials did not differ in the other quartiles (all $p$ values $>0.5)$. The main effect of gaze was not significant $\left(F_{(1,9)}=1.21\right.$, $p=0.30)$.

Thus, these analyses based on a subset of 10 objectively and subjectively unaware participants confirm our main findings observed in the whole set of participants ruling out the possibility that discrepancies between-subjective confidence reports and task performance in some participants may have biased our findings.

\section{Discussion}

We investigated how gaze direction affects the relationship between neural responses to face stimuli and access to awareness 


\section{FFA}
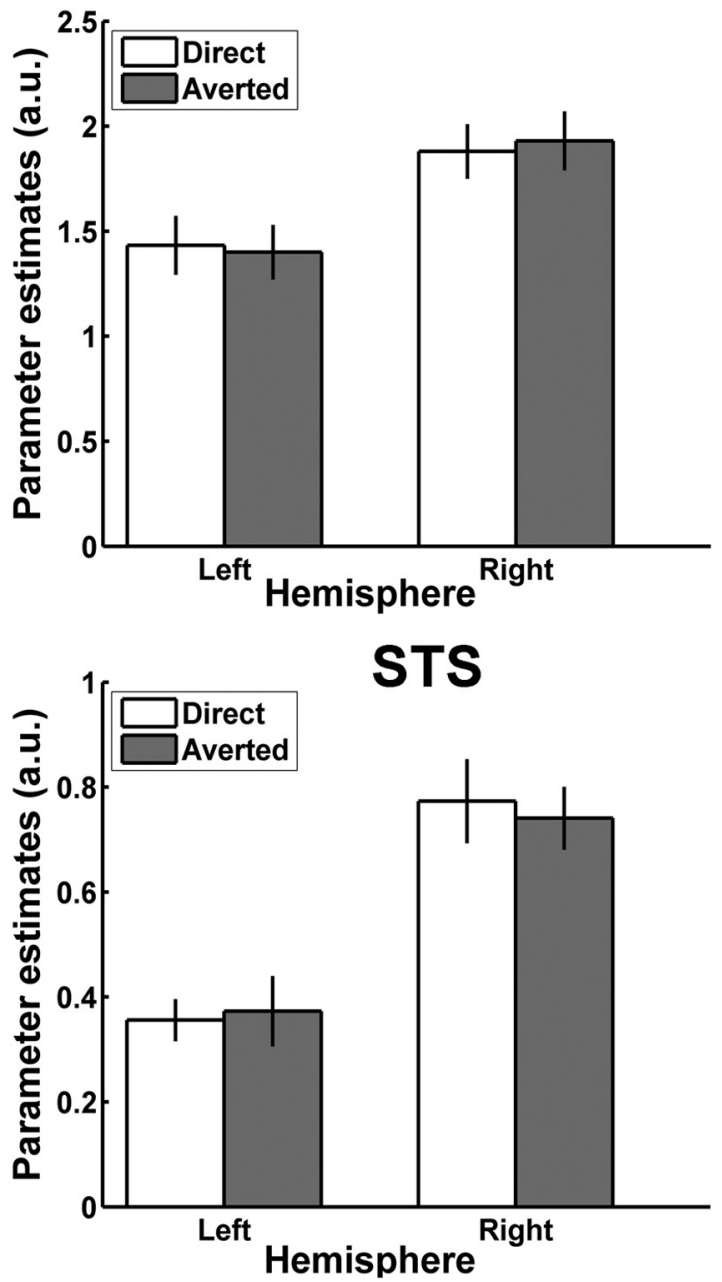

Amygdala
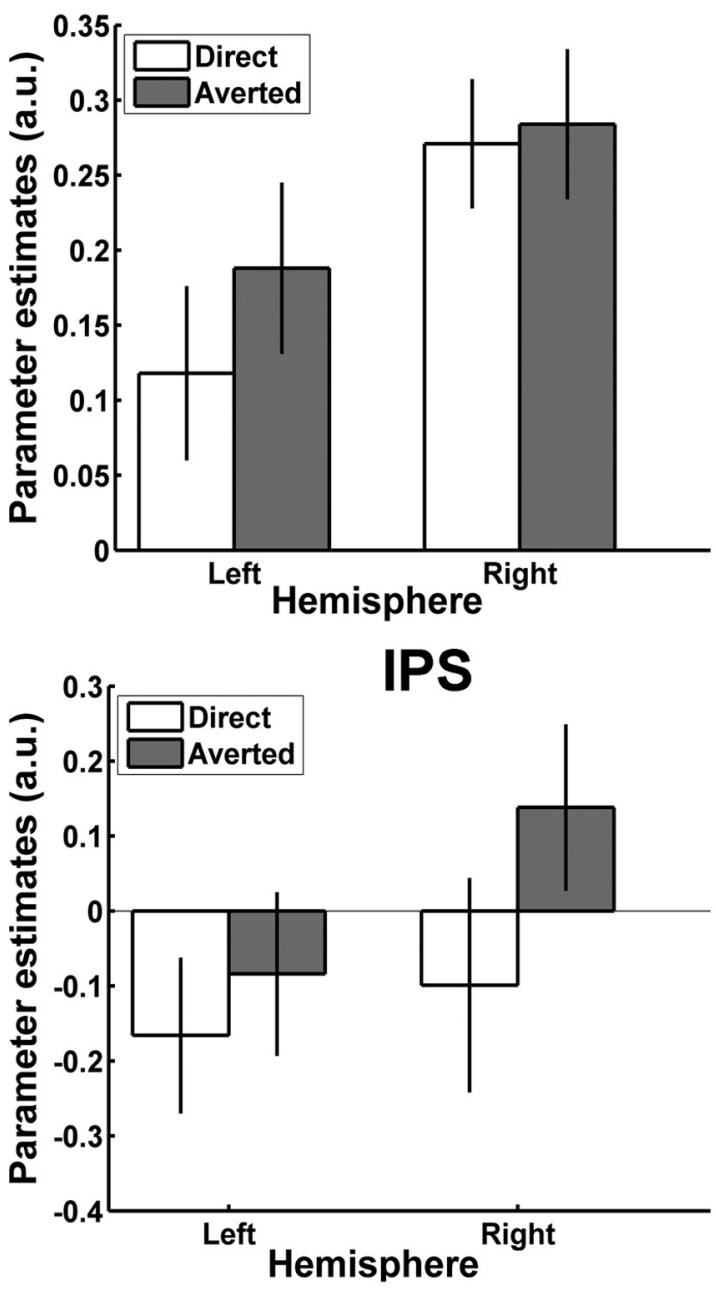

Figure 6. Neural responses to faces with direct and averted gaze in the no-CFS condition for the four regions of interest: FFA, amygdala, STS, and IPS. Error bars denote within-subject SEM (Cousineau, 2005).

under CFS. The FFA, STS, IPS, and amygdala, brain regions known to be involved in gaze processing, showed substantially reduced responses when participants reported to be unaware of the stimuli, consistent with previous findings (for review, see Sterzer et al., 2014). Interestingly, greater neural responses to direct versus averted gaze were observed when participants indicated awareness of the stimuli, whereas responses to unaware direct gaze faces were smaller. This pattern was found to be due to a modulation of the relationship between neural responses and awareness by gaze direction: with increasing neural activation in the FFA, faces with direct gaze entered awareness more readily than faces with averted gaze.

Our finding of enhanced responses to masked but at least partly visible direct-gaze faces is consistent with previous studies investigating gaze-related neural responses to visible faces. For example, in a gender-categorization task using foveally presented faces, FFA activity was stronger for direct versus averted gaze (George et al., 2001). Similarly, the amygdala and STS elicit larger neural responses to direct-gaze faces (Kawashima et al., 1999; George et al., 2001; Pelphrey et al., 2004). Using EEG, alpha band activity, which correlates inversely with vigilance, was found to be reduced and event-related potentials increased for direct versus averted gaze (Gale et al., 1975; Conty et al., 2007). Concurrent with these earlier reports our findings support the notion that the behavioral prioritization of direct gaze, possibly due to increased deployment of attention, is reflected by enhanced neural processing (von Grünau and Anston, 1995; Senju and Hasegawa, 2005).

The special status of eye gaze information for behavior becomes particularly evident by observers' responses to gaze directions in relation to visual awareness: Others' eye gaze not only biases attention in the absence of awareness (Sato et al., 2007; Rothkirch et al., 2015), but direct eye gaze also has the potency to enter awareness more readily than averted gaze (Stein et al., 2011; Chen and Yeh, 2012). Although there is also neural evidence for unconscious processing of gaze direction from previous studies (Burra et al., 2013; Yokoyama et al., 2013), such differential neural responses do not reveal the neural basis for the prioritized access of direct gaze to awareness. By keeping all stimulus parameters constant throughout our experiment, the trial-by-trial subjective experience of the face stimuli reflected the variation of internal representations, rather than the variation of stimulus properties (Hesselmann et al., 2011). This allowed us to directly establish a link between neural responses and access to awareness as a function of gaze direction, independently of other stimulusrelated factors. 
Whereas we found greater neural responses for direct versus averted gaze when participants were aware of faces, we observed the opposite pattern for unaware faces. We surmised that this pattern might be accounted for by the differential effects of gazerelated neural responses on access to awareness. Indeed, behaviorally faces with direct gaze reached participants' awareness more often than faces with averted gaze, in line with previous research (Stein et al., 2011; Chen and Yeh, 2012). Critically, we found that the behavioral sensitivity for direct gaze, indexed by a smaller proportion of direct-gaze faces that remained unconscious, was directly related to the reduced activity levels observed for direct gaze in unaware trials, especially in the FFA. Together with our trial-by-trial analyses showing that a greater proportion of direct-gaze trials reached awareness at similar levels of FFA activity, this suggests that the neural threshold for entering awareness is lower for direct than for averted-gaze faces. Neural signal increases in visual cortex as a function of awareness or stimulus recognition have been shown for a variety of visual stimuli (Grill-Spector et al., 2000; Bar et al., 2001; Moutoussis and Zeki, 2002; Pessoa et al., 2006). Here we provided evidence that this relationship between neural activity and awareness is modulated by a physically subtle but socially highly relevant cue.

The observation that eye gaze modulates the relation between neural activity and awareness raises the question which mechanisms facilitate such access to awareness. One possibility is the existence of neuronal populations or brain regions that are particularly sensitive to the salience of direct gaze and exhibit enhanced responses even in the absence of awareness, thereby facilitating access to awareness at lower levels of neural activity in face-responsive brain regions. However, we found no evidence for such a mechanism, as no brain regions showed greater neural activity in response to direct versus averted face stimuli in unaware trials. Alternatively, there could be qualitative differences in the neural coding of eye-gaze directions in face-responsive brain areas. For example, the neural coding of direct versus averted gaze may be more efficient, e.g., through sparseness (Reddy and Kanwisher, 2006). Such sparser coding may be associated with less metabolic cost and consequently lower BOLD signals, but at the same time ease the readout by other brain areas and thereby facilitate access to awareness (Guyonneau et al., 2004; Olshausen and Field, 2004). However, such an interpretation must currently remain speculative.

The conclusions drawn from our current findings must naturally be limited to the domain of eye-gaze processing. However, our findings provide new insights into the low-level sensory mechanisms underlying the perception of socially relevant stimuli and may thus form the basis for a better understanding of the neural processes governing social interactions on a more general level. Moreover, the observation that highly relevant stimuli require less neural activity to enter awareness, as reported here for direct gaze, may reflect a general mechanism. Whether this mechanism extends to other domains involving ecologically relevant stimuli, e.g., those of emotional or motivational relevance is an intriguing question for future research. It should also be taken into account that our findings are based on the use of CFS, which is just one of several methods available in the laboratory setting to suppress visual stimuli from awareness. As pointed out previously, however, interocular suppression itself also occurs under natural viewing conditions (Arnold, 2011; O'Shea, 2011), thus rendering the method ecologically relevant. Moreover, CFS is well suited and now widely used to measure the potency of visual stimuli to gain access to awareness (Gayet et al., 2014; Stein and Sterzer, 2014) and its use in the current fMRI study allows us to directly relate our neural findings to earlier behavioral reports (Stein et al., 2011; Chen and Yeh, 2012).

In addition to CFS, our experiment comprised a control condition where faces were presented fully visible without CFS masks. This condition was included to verify activation in our ROIs to face stimuli. Whereas the presentation of visible faces led to substantial activations in these areas, gaze-specific effects in this condition were only detectable after substantially lowering the statistical threshold. Although the absence of differential effects at a more conservative threshold seems to be in conflict with previous work (George et al., 2001; Pelphrey et al., 2004; Schilbach et al., 2006), critical differences between our and previous studies have to be noted. In our study, gaze directions were neither task-relevant nor were participants informed about them beforehand. Furthermore, we presented static faces, whereas the brain regions of the gaze-processing network respond more strongly to dynamic faces (Pelphrey et al., 2004; Schilbach et al., 2006).

But why then did visible faces under CFS entail differential neural responses to eye gaze? Full visibility of face stimuli likely involves strong feedback signals from other brain areas, boosting activity in gaze processing regions. Because gaze direction only represents a minor, though relevant feature of the whole face, neural activity may predominantly be driven by holistic face processing. Thus, the absence of gaze-specific effects in trials without CFS masks may reflect a ceiling effect, where differences in eye gaze have a minor impact relative to the processing of the whole face. In contrast, the concurrent presentation of CFS stimuli may have mitigated the overall response strength through neural competition induced by interocular suppression, thereby increasing the sensitivity for subtle gaze-related neural response differences. It is important to note that our main finding in this study results from the comparison between aware and unaware trials with respect to differences between direct and averted gaze processing under CFS. Such a comparison cannot be performed in the noCFS control condition due to the absence of unaware trials. Furthermore, our aim was to investigate the relationship between neural responses to gaze direction and access to awareness, rather than investigating differential responses to gaze under awareness. Thus, the absence of gaze-specific effects in the no-CFS condition which was only included to verify activation to faces in our ROIs does not weaken our main conclusion.

To conclude, we show that gaze direction modulates the relationship between neural activity and access to awareness. Critically, we were able to establish a link between neural responses to face stimuli and their access to awareness by showing that direct gaze, a salient social stimulus, requires less neural activity in faceresponsive brain regions to reach awareness than averted gaze.

\section{References}

Arnold DH (2011) Why is binocular rivalry uncommon? Discrepant monocular images in the real world. Front Hum Neurosci 5:116. CrossRef Medline

Bar M, Tootell RB, Schacter DL, Greve DN, Fischl B, Mendola JD, Rosen BR, Dale AM (2001) Cortical mechanisms specific to explicit visual object recognition. Neuron 29:529-535. CrossRef Medline

Bristow D, Rees G, Frith CD (2007) Social interaction modifies neural response to gaze shifts. Soc Cogn Affect Neurosci 2:52-61. CrossRef Medline

Burra N, Hervais-Adelman A, Kerzel D, Tamietto M, de Gelder B, Pegna AJ (2013) Amygdala activation for eye contact despite complete cortical blindness. J Neurosci 33:10483-10489. CrossRef Medline

Carlin JD, Calder AJ, Kriegeskorte N, Nili H, Rowe JB (2011) A head viewinvariant representation of gaze direction in anterior superior temporal sulcus. Curr Biol 21:1817-1821. CrossRef Medline 
Chen YC, Yeh SL (2012) Look into my eyes and I will see you: unconscious processing of human gaze. Conscious Cogn 21:1703-1710. CrossRef Medline

Conty L, Tijus C, Hugueville L, Coelho E, George N (2006) Searching for asymmetries in the detection of gaze contact versus averted gaze under different head views: a behavioural study. Spat Vis 19:529-545. CrossRef Medline

Conty L, N'Diaye K, Tijus C, George N (2007) When eye creates the contact! ERP evidence for early dissociation between direct and averted gaze motion processing. Neuropsychologia 45:3024-3037. CrossRef Medline

Cousineau D (2005) Confidence intervals in within-subject designs: a simpler solution to Loftus and Masson's method. TQMP 1:42-45.

Dienes Z (2011) Bayesian versus orthodox statistics: which side are you on? Perspect Psychol Sci 6:274-290. CrossRef Medline

Emery NJ (2000) The eyes have it: the neuroethology, function and evolution of social gaze. Neurosci Biobehav Rev 24:581-604. CrossRef Medline

Engell AD, Haxby JV (2007) Facial expression and gaze-direction in human superior temporal sulcus. Neuropsychologia 45:3234-3241. CrossRef Medline

Ethofer T, Gschwind M, Vuilleumier P (2011) Processing social aspects of human gaze: a combined fMRI-DTI study. Neuroimage 55:411-419. CrossRef Medline

Gale A, Spratt G, Chapman AJ, Smallbone A (1975) EEG correlates of eye contact and interpersonal distance. Biol Psychol 3:237-245. CrossRef Medline

Gayet S, Van der Stigchel S, Paffen CL (2014) Breaking continuous flash suppression: competing for consciousness on the pre-semantic battlefield. Front Psychol 5:460. CrossRef Medline

George N, Driver J, Dolan RJ (2001) Seen gaze-direction modulates fusiform activity and its coupling with other brain areas during face processing. Neuroimage 13:1102-1112. CrossRef Medline

Grill-Spector K, Kushnir T, Hendler T, Malach R (2000) The dynamics of object-selective activation correlate with recognition performance in humans. Nat Neurosci 3:837-843. CrossRef Medline

Grosbras MH, Laird AR, Paus T (2005) Cortical regions involved in eye movements, shifts of attention, and gaze perception. Hum Brain Mapp 25:140-154. CrossRef Medline

Guyonneau R, Vanrullen R, Thorpe SJ (2004) Temporal codes and sparse representations: a key to understanding rapid processing in the visual system. J Physiol Paris 98:487-497. CrossRef Medline

Hadjikhani N, Hoge R, Snyder J, de Gelder B (2008) Pointing with the eyes: the role of gaze in communicating danger. Brain Cogn 68:1-8. CrossRef Medline

Haxby JV, Hoffman EA, Gobbini MI (2002) Human neural systems for face recognition and social communication. Biol Psychiatry 51:59-67. CrossRef Medline

Hesselmann G, Hebart M, Malach R (2011) Differential BOLD activity associated with subjective and objective reports during "blindsight" in normal observers. J Neurosci 31:12936-12944. CrossRef Medline

Hoffman EA, Haxby JV (2000) Distinct representations of eye gaze and identity in the distributed human neural system for face perception. Nat Neurosci 3:80-84. CrossRef Medline

Hooker CI, Paller KA, Gitelman DR, Parrish TB, Mesulam MM, Reber PJ (2003) Brain networks for analyzing eye gaze. Brain Res Cogn Brain Res 17:406-418. CrossRef Medline

Horley K, Williams LM, Gonsalvez C, Gordon E (2003) Social phobics do not see eye to eye: a visual scanpath study of emotional expression processing. J Anxiety Disord 17:33-44. CrossRef Medline

Kampe KK, Frith CD, Dolan RJ, Frith U (2001) Psychology: reward value of attractiveness and gaze. Nature 413:589. CrossRef Medline

Kato C, Matsuo K, Matsuzawa M, Moriya T, Glover GH, Nakai T (2001) Activation during endogenous orienting of visual attention using symbolic pointers in the human parietal and frontal cortices: a functional magnetic resonance imaging study. Neurosci Lett 314:5-8. CrossRef Medline

Kawashima R, Sugiura M, Kato T, Nakamura A, Hatano K, Ito K, Fukuda H, Kojima S, Nakamura K (1999) The human amygdala plays an important role in gaze monitoring: a PET study. Brain 122:779-783. CrossRef Medline

Kleinke CL (1986) Gaze and eye contact: a research review. Psychol Bull 100:78-100. CrossRef Medline

Kleinke CL, Singer DA (1979) Influence of gaze on compliance with de- manding and conciliatory requests in a field setting. Pers Soc Psychol Bull 5:386-390. CrossRef

Leopold DA, Wilke M, Maier A, Logothetis NK (2002) Stable perception of visually ambiguous patterns. Nat Neurosci 5:605-609. CrossRef Medline

Macrae CN, Hood BM, Milne AB, Rowe AC, Mason MF (2002) Are you looking at me? Eye gaze and person perception. Psychol Sci 13:460-464. CrossRef Medline

Mason MF, Hood BM, Macrae CN (2004) Look into my eyes: gaze direction and person memory. Memory 12:637-643. CrossRef Medline

Materna S, Dicke PW, Thier P (2008) Dissociable roles of the superior temporal sulcus and the intraparietal sulcus in joint attention: a functional magnetic resonance imaging study. J Cogn Neurosci 20:108-119. CrossRef Medline

Mosconi MW, Mack PB, McCarthy G, Pelphrey KA (2005) Taking an "intentional stance" on eye-gaze shifts: a functional neuroimaging study of social perception in children. Neuroimage 27:247-252. CrossRef Medline

Moutoussis K, Zeki S (2002) The relationship between cortical activation and perception investigated with invisible stimuli. Proc Natl Acad Sci U S A 99:9527-9532. CrossRef Medline

Nummenmaa L, Calder AJ (2009) Neural mechanisms of social attention. Trends Cogn Sci 13:135-143. CrossRef Medline

Nummenmaa L, Passamonti L, Rowe J, Engell AD, Calder AJ (2010) Connectivity analysis reveals a cortical network for eye gaze perception. Cereb Cortex 20:1780-1787. CrossRef Medline

Nummenmaa L, Engell AD, von dem Hagen E, Henson RN, Calder AJ (2012) Autism spectrum traits predict the neural response to eye gaze in typical individuals. Neuroimage 59:3356-3363. CrossRef Medline

Olshausen BA, Field DJ (2004) Sparse coding of sensory inputs. Curr Opin Neurobiol 14:481-487. CrossRef Medline

O'Shea RP (2011) Binocular rivalry stimuli are common but rivalry is not. Front Hum Neurosci 5:148. CrossRef Medline

Pearson J, Brascamp J (2008) Sensory memory for ambiguous vision. Trends Cogn Sci 12:334-341. CrossRef Medline

Pelphrey KA, Singerman JD, Allison T, McCarthy G (2003) Brain activation evoked by perception of gaze shifts: the influence of context. Neuropsychologia 41:156-170. CrossRef Medline

Pelphrey KA, Viola RJ, McCarthy G (2004) When strangers pass processing of mutual and averted social gaze in the superior temporal sulcus. Psychol Sci 15:598-603. CrossRef Medline

Pelphrey KA, Morris JP, McCarthy G (2005) Neural basis of eye gaze processing deficits in autism. Brain 128:1038-1048. CrossRef Medline

Pessoa L, Japee S, Sturman D, Ungerleider LG (2006) Target visibility and visual awareness modulate amygdala responses to fearful faces. Cereb Cortex 16:366-375. CrossRef Medline

Puce A, Allison T, Bentin S, Gore JC, McCarthy G (1998) Temporal cortex activation in humans viewing eye and mouth movements. J Neurosci 18:2188-2199. Medline

Reddy L, Kanwisher N (2006) Coding of visual objects in the ventral stream. Curr Opin Neurobiol 16:408-414. CrossRef Medline

Rothkirch M, Madipakkam AR, Rehn E, Sterzer P (2015) Making eye contact without awareness. Cognition 143:108-114. CrossRef Medline

Sato W, Okada T, Toichi M (2007) Attentional shift by gaze is triggered without awareness. Exp Brain Res 183:87-94. CrossRef Medline

Sato W, Kochiyama T, Uono S, Yoshikawa S (2008) Time course of superior temporal sulcus activity in response to eye gaze: a combined fMRI and MEG study. Soc Cogn Affect Neurosci 3:224-232. CrossRef Medline

Sato W, Kochiyama T, Uono S, Yoshikawa S (2009) Commonalities in the neural mechanisms underlying automatic attentional shifts by gaze, gestures, and symbols. Neuroimage 45:984-992. CrossRef Medline

Sauer A, Mothes-Lasch M, Miltner WH, Straube T (2014) Effects of gaze direction, head orientation and valence of facial expression on amygdala activity. Soc Cogn Affect Neurosci 9:1246-1252. CrossRef Medline

Schilbach L, Wohlschlaeger AM, Kraemer NC, Newen A, Shah NJ, Fink GR, Vogeley K (2006) Being with virtual others: neural correlates of social interaction. Neuropsychologia 44:718-730. CrossRef Medline

Schurger A (2009) A very inexpensive MRI-compatible method for dichoptic visual stimulation. J Neurosci Methods 177:199-202. CrossRef Medline 
Senju A, Hasegawa T (2005) Direct gaze captures visuospatial attention. Vis Cogn 12:127-144. CrossRef

Senju A, Johnson MH (2009) The eye contact effect: mechanisms and development. Trends Cogn Sci 13:127-134. CrossRef Medline

Senju A, Tojo Y, Yaguchi K, Hasegawa T (2005) Deviant gaze processing in children with autism: an ERP study. Neuropsychologia 43:1297-1306. CrossRef Medline

Snyder M, Grather J, Keller K (1974) Staring and compliance: a field experiment on hitchhiking. J Appl Soc Psychol 4:165-170. CrossRef

Stein T, Sterzer P (2014) Unconscious processing under interocular suppression: getting the right measure. Front Psychol 5:387. CrossRef Medline

Stein T, Senju A, Peelen MV, Sterzer P (2011) Eye contact facilitates awareness of faces during interocular suppression. Cognition 119:307-311. CrossRef Medline

Sterzer P, Rees G (2008) A neural basis for percept stabilization in binocular rivalry. J Cogn Neurosci 20:389-399. CrossRef Medline

Sterzer P, Haynes JD, Rees G (2008) Fine-scale activity patterns in high-level visual areas encode the category of invisible objects. J Vis 8(15):10 1-12. CrossRef Medline
Sterzer P, Stein T, Ludwig K, Rothkirch M, Hesselmann G (2014) Neural processing of visual information under interocular suppression: a critical review. Front Psychol 5:453. CrossRef Medline

Straube T, Langohr B, Schmidt S, Mentzel HJ, Miltner WH (2010) Increased amygdala activation to averted versus direct gaze in humans is independent of valence of facial expression. Neuroimage 49:2680-2686. CrossRef Medline

Szczepanowski R, Traczyk J, Wierzchon M, Cleeremans A (2013) The perception of visual emotion: comparing different measures of awareness. Conscious Cogn 22:212-220. CrossRef Medline

Tsuchiya N, Koch C (2005) Continuous flash suppression reduces negative afterimages. Nat Neurosci 8:1096-1101. CrossRef Medline

von Grünau M, Anston C (1995) The detection of gaze direction: a stare-inthe-crowd effect. Perception 24:1297-1313. CrossRef Medline

Yokoyama T, Noguchi Y, Kita S (2013) Unconscious processing of direct gaze: evidence from an ERP study. Neuropsychologia 51:1161-1168. CrossRef Medline

Zehetleitner M, Rausch M (2013) Being confident without seeing: what subjective measures of visual consciousness are about. Atten Percept Psychophys 75:1406-1426. CrossRef Medline 